\title{
Target vibration estimation in SAR based on phase-analysis method
}

\author{
Weijie Xia ${ }^{*}$ (D) and Linlin Huang
}

\begin{abstract}
An efficient vibration estimation method for synthetic aperture radar (SAR) systems based on phase-analysis method is presented. Small vibrations, which consist of multiple frequency components and are usually rather weak, introduce phase modulation in radar echoes. They contain important signatures of objects. Extracting and analyzing the phase from radar echoes is one of the significant approaches to acquire vibration characteristics. In order to separate different targets in different range cells, the proposed method acts the range compression and range migration correction on the radar echoes, followed by extracting the phase history of each target's radar echoes. Since the phase history contains the time-varying ranges from the targets to airborne radar besides the vibration signal, we apply wavelet transform to the phase history to extract the vibration signal. Then the vibration signatures would be estimated quantitatively. The method is a more efficient and convenient approach to estimate the vibration amplitudes and frequencies of one or more targets, whose vibration signal is a single-frequency or multi-frequency signal. Moreover, it can be applied in the case where the airborne radar moves along the desirable or undesirable trajectory. Simulation results indicate that this method provides a larger range of estimable vibration frequency, higher estimation precision, and lower computation complexity.
\end{abstract}

Keywords: Linear frequency modulated (LFM) signal, Phase analysis, Vibration, Wavelet transform (WT)

\section{Introduction}

Vibration is ubiquitous in the real world. Objects' vibration signatures bear vital information about the type of the objects [1]. Spaceborne synthetic aperture radar (SAR) can operate over thousands of kilometers, which is an effective way to acquire vibration signatures of structures placed in remote locations. Small vibrations introduce phase modulation in the returned SAR signals, which is often referred to as the micro-Doppler effect [2]. By analyzing the micro-Doppler effect, we can identify and classify the objects according to the vibration signatures. In addition, the vibration signatures can be used to compensate the phase errors in SAR returns to eliminate the image blur $[3,4]$. Therefore, target vibration signatures estimation has great significance.

Several methods have been presented in the literature which deal with the problem of how to extract the vibration signature with SAR systems. The phase-modulated

\footnotetext{
* Correspondence: nuaaxwj@nuaa.edu.cn

Key Laboratory of Radar Imaging and Microwave Photonics (Nanjing Univ. Aeronaut. Astronaut.), Ministry of Education, College of Electronic and Information Engineering, Nanjing University of Aeronautics and Astronautics, Nanjing 210016, China
}

signals induced by vibration with Cohen's class time-frequency method were analyzed, and the time-frequency signatures and the SAR image of a vibrating target were studied in [5-7]. In [8] and [9], the Doppler frequency of the rotating and vibrating targets was analyzed with smoothed pseudo Wigner-Ville distribution. A novel vibration estimation method based on the discrete fractional Fourier transform (DFRFT) was presented in [10-12]. The method in [13] with sinusoidal frequency modulation Fourier transform (SFMFT) was proposed to obtain the frequency spectrum of vibration traces. In [14], the target was processing with autofocus to estimate the phase error caused by vibration, and the vibration parameters were calculated based on the phase error. Another extracting method of vibrating features based on slow time envelope (STE) signatures was presented in [15]. In [16] and [17], a micro-Doppler reconstruction method using azimuth time-frequency tracking of the phase history was proposed.

In this paper, a vibration estimation method using SAR is presented based on phase analysis. In comparison with the previous works, our approach differs in two 
main ways. On the one hand, this method is applicable to the case where a single scatterer vibrating in multiple frequencies and several scatterers distributed in different range cells are placed within a SAR scene. In addition, it is also suitable for the situation where the spaceborne radar moves along the undesirable trajectory. On the other hand, compared to methods which rely on the interpretation of time-frequency representations, the proposed method provides a quantitative estimation of the vibration signatures by offering the history of the instantaneous displacement and the spectrum of the vibrating object. The estimation results contain complete parameters, not only the vibration amplitudes and frequencies but also the initial phases included.

The procedure of this method is as follows. It starts with the conventional SAR processing procedure to obtain a nonstationary signal from the vibrating target. First, the returned SAR signals are demodulated. Second, range compression is acted on the demodulated SAR echoes, followed by an application of range cell migration correction to range lines. Hence, the signal from a vibrating target is focused on a range line. Since small vibrations modulate the phase in radar echoes, we extract the phase history of each range line containing vibrating objects and also analyze the restrictive condition of phase ambiguity. Next, by performing the wavelet transform, the vibration displacement is acquired and vibration amplitudes and frequencies of each target are estimated quantitatively. The influence of noises on the performance of the proposed approach is also discussed. The method is capable of extracting vibration signatures in the case where the airborne radar moves along the desirable or undesirable trajectory. Finally, the simulation is given and this method has been compared with other radar-based vibration estimation method. The results illustrate the superior of this method.

The remainder of this paper is organized as follows. In Section 2, the motion model is established and the mathematical formulas of SAR returns containing vibration signatures are derived. In Section 3, the phase-analysis algorithm including phase extraction and wavelet transform is introduced, followed by the discussion related to the signal-to-noise ratio (SNR) in Section 4. Simulations are provided and a comparison between the proposed method and some of others is presented in Section 5. Section 6 gives the conclusion.

\section{Signal model}

Figure 1 shows a 3-D SAR flight geometry. Aircraft with the airborne radar sensor flies along the axis $O X$ from the point $A$ to $B$, whose instantaneous location is $(x(\eta)$, $y(\eta), h)$, where $\eta$ is the azimuth time. The vibrating target is located at the point $C\left(0, y_{0}, 0\right)$. The nominal line-ofsight distance from the target to the radar sensor is

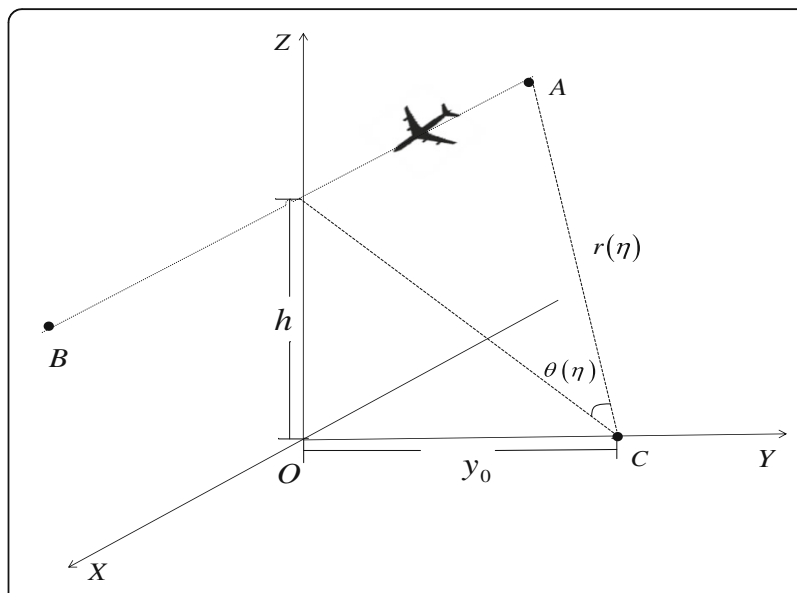

Fig. 1 Three-dimensional SAR flight geometry

$$
r(\eta)=\sqrt{x^{2}(\eta)+\left(y(\eta)-y_{0}\right)^{2}+h^{2}}
$$

Let $r_{v}(\eta)$ denote the projection of the vibration displacement onto the line of sight (LOS) from the radar sensor to the target. The instantaneous slant range becomes

$$
R(\eta)=r(\eta)+r_{\nu}(\eta)=r(\eta)+r_{\nu 0}(\eta) \cdot \cos \theta(\eta)
$$

where $\theta(\eta)$ is the angle between the instantaneous slant range and the shortest distance from the target to the radar sensor and $r_{v 0}$ represents $r_{v}(\eta)$ for $\theta(\eta)=0$. Since the change of $\theta(\eta)$ is usually small, we obtain that $r_{\nu}(\eta) \approx r_{v 0}(\eta)$. Then $R(\eta)$ can be approximated by

$$
R(\eta) \approx r(\eta)+r_{\nu 0}(\eta)
$$

Most vibration signals are usually referred to as a periodic function, which can be expanded in Fourier series. Any vibration that generated in practice satisfies the Dirichlet conditions, and therefore, the Fourier series of vibration signal is convergent [18]. Consequently, the traces of the vibration can be concluded into a series of periodic sinusoids. Here, we assume that the vibration displacement projected onto LOS is modeled by

$$
r_{\nu 0}(\eta)=\sum_{i=1} a_{i} \sin \left(2 \pi f_{v i} \eta+\varphi_{i}\right)
$$

where $a_{i}$ is the projection of the vibration amplitude onto LOS, $f_{v i}$ is the vibration frequency, and $\varphi_{i}$ is the initial phase of the vibration signal.

Consider a side-looking strip-mode SAR, whose transmitting waveform is a chirp signal, with carrier frequency $f_{0}$ and chirp rate $K_{r}$. Each returned SAR pulse is demodulated by the transmitting signal delayed appropriately by the round-trip time to the center of the illuminated patch. A demodulated pulse of a single point target can be written as [19] 


$$
\begin{aligned}
& s_{0}(\tau, \eta)=A_{0} w_{r}\left[\tau-\frac{2 R(\eta)}{c}\right] w_{a}\left[\eta-\eta_{c}\right] \\
& \cdot \exp \left\{-j 4 \pi f_{0} \frac{R(\eta)}{c}\right\} \exp \left\{j \pi K_{r}\left(\tau-\frac{2 R(\eta)}{c}\right)^{2}\right\}
\end{aligned}
$$

where $A_{0}$ is a complex constant, denoting the reflectivity of the target, $c$ is the propagation speed of the pulse, $\tau$ is the range time, $\eta_{c}$ represents zero Doppler time, $w_{r}[\bullet]$ is the envelope of the pulse, usually approximated as a rectangular window and $w_{a}[\bullet]$ is the radiation pattern of the radar antenna. If there are more than one point targets, the SAR returns become

$$
\begin{aligned}
s_{0}(\tau, \eta)= & \sum_{n=1}^{N} A_{0 n} w_{r}\left[\tau-\frac{2 R_{n}(\eta)}{c}\right] w_{a}\left[\eta-\eta_{c}\right] \\
& \cdot \exp \left\{-j 4 \pi f_{0} \frac{R_{n}(\eta)}{c}\right\} \exp \left\{j \pi K_{r}\left(\tau-\frac{2 R_{n}(\eta)}{c}\right)^{2}\right\}
\end{aligned}
$$

According to (3), (4), and (6), we know that the small range perturbation of vibrating targets modulates the phase term in radar echo, which is referred to as the micro-Doppler effect. Figure 2 shows a reconstructed SAR image of a vibrating target. Due to the microDoppler effect, azimuth compression cannot focus the vibrating scatterer on the correct cross-range position, with some significant sidelobes nearby, which is commonly called ghost targets [20]. Consequently, we can extract some information related to micro-motion by analyzing the phase of the SAR returns.

\section{Algorithm}

\subsection{Phase extraction}

Range compression is applied to the SAR returned signal to separate the scatterers in range. By performing the

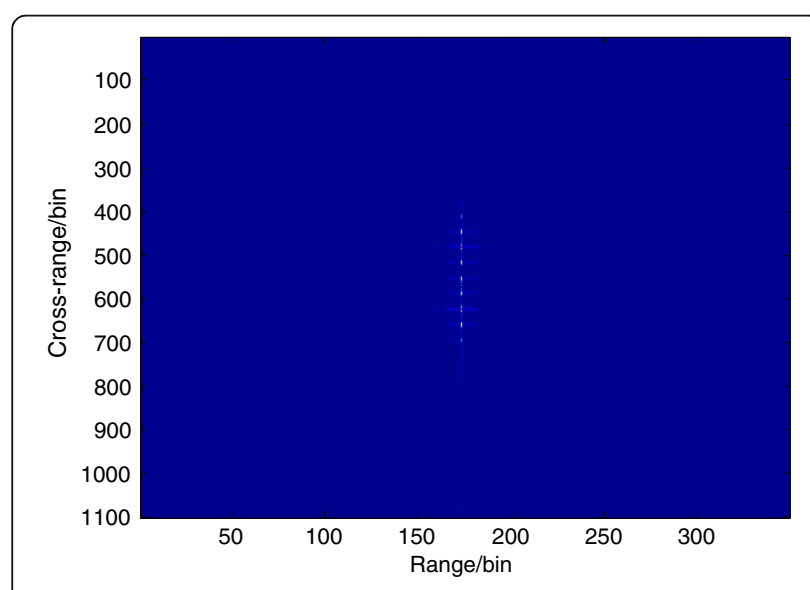

Fig. 2 SAR image of a vibrating point target matching filtering in frequency domain, we get the result of the range compression

$$
\begin{aligned}
s_{r c}(\tau, \eta)= & \sum_{n=1}^{N} A_{0 n} p_{r}\left[\tau-2 R_{n}(\eta) / c\right] w_{a}\left(\eta-\eta_{c}\right) \\
& \cdot \exp \left\{-j 4 \pi f_{0} R_{n}(\eta) / c\right\}
\end{aligned}
$$

where $p_{r}[\bullet]$ is the a sinc function. Then the range migration correction algorithm can be applied to (7) to correct for range cell migration, the signal becomes

$$
\begin{aligned}
s_{r c}{ }^{\prime}(\tau, \eta) & =\sum_{n=1}^{N} s_{r c n}{ }^{\prime}(\tau, \eta) \\
& =\sum_{n=1}^{N} A_{0 n} p_{r}\left[\tau-2 R_{0 n} / c\right] w_{a}\left(\eta-\eta_{c}\right) \\
& \cdot \exp \left\{-j 4 \pi f_{0} R_{n}(\eta) / c\right\}
\end{aligned}
$$

where $R_{0 n}$ is the distance from $n$th point target to the midaperture. Now, the range-compressed phase history of one point target is in the same range cell. The phase of the range line containing $n$th target can be solved by

$$
\phi_{n}(\eta)=\left.\arg \left[s_{\mathrm{rcn}}{ }^{\prime}(\tau, \eta)\right]\right|_{\tau=2 R_{0 n}} / c
$$

i.e.,

$$
\phi_{n}(\eta)=-4 \pi f_{0} R_{n}(\eta) / c
$$

Figure 3 shows the range lines of two vibrating targets, which can be obtained by applying range compression and range migration correction to the SAR returns. The two point targets are separated in range.

Since the measured phase is limited only between $-\pi$ and $\pi$, the phase ambiguity occurs when some values of the real phase $-4 \pi f_{0} R_{n}(\eta) / c$ are over this range. The phase total shift between the measured phase and the real phase is $n$ times of $2 \pi$, where $n$ is an integer to be determined. Fortunately, this can be corrected by comparing the

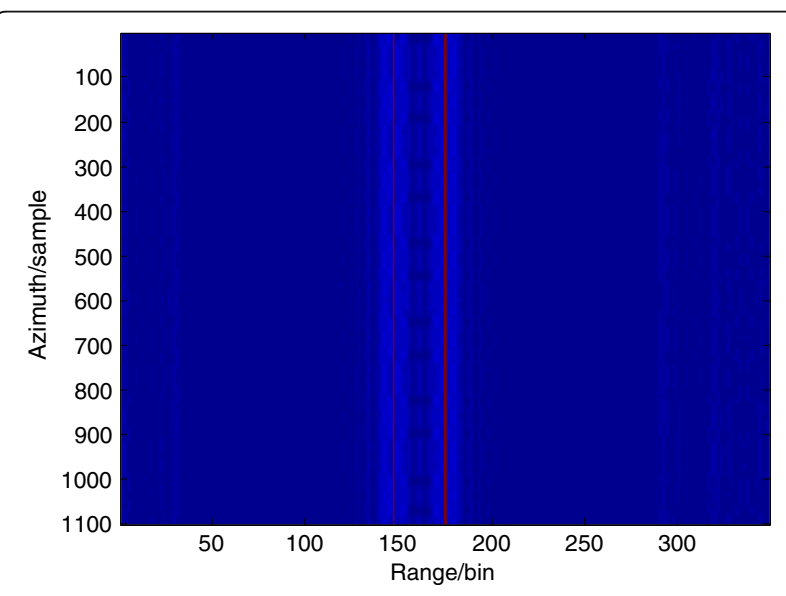

Fig. 3 Range lines of two vibrating targets 
adjacent samples. Supposing the maximal real phase difference between adjacent samples is below $\pi$, a solution of the phase revising function is

$$
\operatorname{pr}\left(\eta_{k}\right)= \begin{cases}\operatorname{pr}\left(\eta_{k-1}\right)-2 \pi, & \phi_{n}\left(\eta_{k}\right)-\phi_{n}\left(\eta_{k-1}\right)>\pi \\ \operatorname{pr}\left(\eta_{k-1}\right)+2 \pi, & \phi_{n}\left(\eta_{k-1}\right)-\phi_{n}\left(\eta_{k}\right)>\pi \\ \operatorname{pr}\left(\eta_{k-1}\right), & \left|\phi_{n}\left(\eta_{k}\right)-\phi_{n}\left(\eta_{k-1}\right)\right|<\pi\end{cases}
$$

where $\operatorname{pr}\left(\eta_{k}\right)$ is the phase total shift between the real phase and measurements at $k$ th azimuth time. Thus, the revised phase can be expressed as

$$
\phi_{n}{ }^{\prime}(\eta)=\phi_{n}(\eta)+\operatorname{pr}(\eta)
$$

However, the discrete signal is subjected to a problem. If the real phase difference between adjacent samples is greater than $\pi$, we cannot acquire the correct real phase by above phase-revising method. For example, in one case, if the adjacent samples' real phase difference is greater than $2 \pi$, like point 2 and 3 in Fig. $4 \mathrm{a}$, the phase shift between the real phase and the measured phase of point 3 is also larger than $2 \pi$ (actually the phase shift equals $4 \pi$ here). By applying the above method, we only add $2 \pi$ to the measured phase (point 3') to obtain the revised phase (point 3 "), which is not equal to the real phase (point 3). In another case, as shown in Fig. 4b, the adjacent samples' real phase, like point 5 and 6 , whose difference is smaller than $2 \pi$ but larger than $\pi$, so that the phase difference between their measured phase (point $5^{\prime}$ and 6 ) is below $\pi$. With application of the above phase-revising method, the

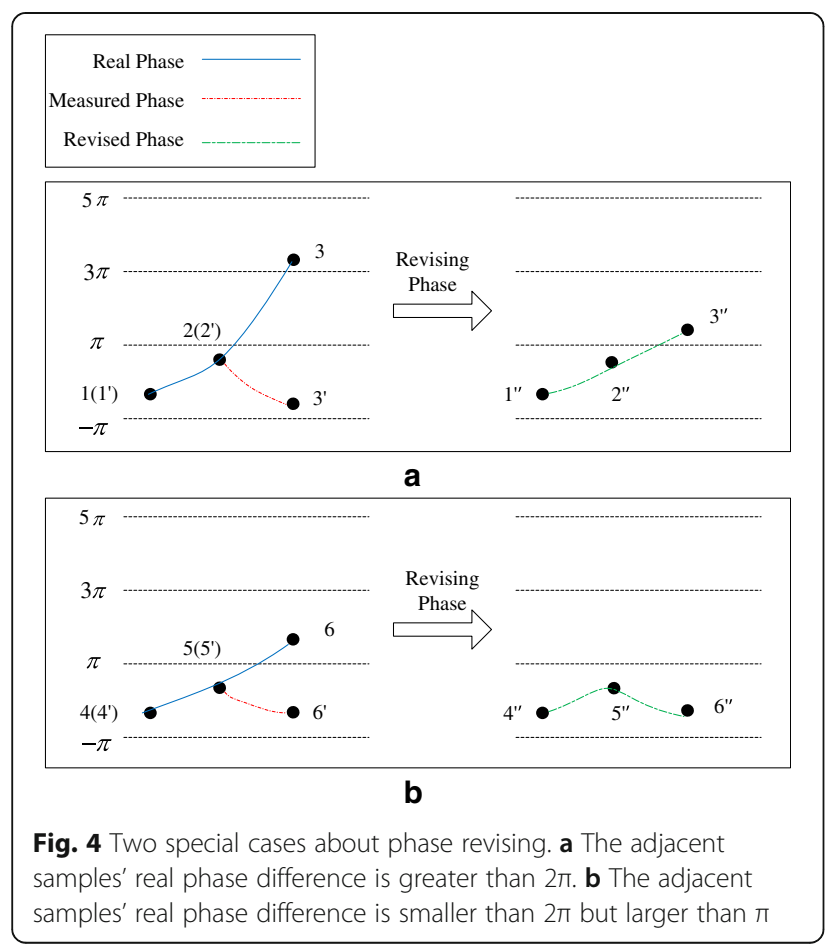

revised phase (point $6 "$ ) is equal to the measured phase (point 6 ), but not the real phase (point 6). In order to avoid the occurrence of the above, we need to increase pulse repetition frequency (PRF) to make sure that the real phase difference between adjacent samples is below $\pi$. Therefore, the limitation of PRF has to be addressed here.

First, we consider the situation where the airborne radar moves in a straight line at a constant speed, and suppose that there is only one vibrating target whose vibration displacement along the direction of radar incidence is $a \sin \left(2 \pi f_{v} \eta\right)$. Let $f_{p r f}$ denote PRF, and the sampling interval of slow time is

$$
\Delta \eta=1 / f_{p r f}
$$

Since the maximal phase difference between adjacent samples is always occurred where the slope of vibration displacement is maximal, it can be expressed as

$$
\begin{aligned}
\Delta \phi_{\max } & =\frac{4 \pi f_{0} \cdot 2 a \sin \left(2 \pi f_{v} \cdot \Delta \eta / 2\right)}{c} \\
& =\frac{8 \pi f_{0} a \sin \left(\pi f_{v} \cdot \Delta \eta\right)}{c}
\end{aligned}
$$

Due to $\Delta \phi_{\max }$ must be constrained below $\pi$, i.e., $8 \pi f_{0} a$ $\sin \left(\pi f_{v} \cdot \Delta \eta\right) / c<\pi$, the requirement of PRF can be solved via

$$
f_{\text {prf }}>\frac{\pi f_{v}}{\arcsin \left(\frac{c}{8 a f_{0}}\right)}
$$

According to Nyquist sampling theorem, PRF must be larger than $2 f_{v}$. And for the vibration amplitude meets $a>0, \arcsin \left(\frac{c}{8 a f_{0}}\right)$ should be satisfied as $0<\arcsin$ $\left(\frac{c}{8 a f_{0}}\right)<\frac{\pi}{2}$.

Then, we take another situation into account. The airborne radar does not simply move in a straight trajectory, while it flies with a velocity $v_{Y}$, which is a velocity along axis OY, that leads to the slant range $R(\eta)$ fluctuating with azimuth time. The range of fluctuation is much larger than vibration amplitude so that we can only take fluctuation into consideration. In this case, the maximal phase difference between adjacent samples becomes

$$
\Delta \phi_{\max }=\frac{4 \pi f_{0}\left|v_{Y}\right|_{\max } \cdot \Delta \eta}{c}
$$

According to $\Delta \phi_{\max }<\pi$ and (13), we can get the limiting condition of PRF

$$
f_{\text {prf }}>\frac{4 f_{0}\left|v_{Y}\right|_{\max }}{c}
$$

\subsection{Wavelet transform}

Compared to Fourier transform, wavelet transform (WT) is a local transform in time and frequency domain, which 
can be used to extract information from signal effectively. Wavelet transform makes a multi-scale analysis of functions or signals by expansion, translation operations, so that some characteristics can be fully highlighted. It is known as mathematical microscope.

According to (10), the phase of the range line is $-4 \pi f_{0} R_{n}(\eta) / c$, which can be written in

$$
\phi_{n}(\eta)=-4 \pi f_{0}\left(r(\eta)+r_{\nu 0}(\eta)\right) / c
$$

This expression demonstrates that the phase of the range line is the sum of two parts: $-4 \pi f_{0} \cdot r(\eta) / c$, which is linear proportional to the nominal line-of-sight distance from the target to the radar sensor and $-4 \pi f_{0} \cdot r_{\nu 0}(\eta) / c$. The former varies slowly with azimuth time referred to as a low-frequency signal. The latter induced by vibration is equivalent to a high-frequency signal. Here, we extract the low-frequency signal from the phase of range line by wavelet transform, and the remaining high-frequency signal is the vibration signal that we need. The algorithm chart is shown in Fig. 5.

If we applied Fourier transform to (18) directly, vibration signal would be submerged in the low-frequency signal in spectrogram, as shown in Fig. 6. This is because the changed slant distance is equivalent to a low-frequency signal, whose amplitude is much larger than vibration amplitude. In this way, it is not easy to distinguish the vibration signal and acquire its frequency from the spectrum. Therefore, we have to separate the vibration signal from the low-frequency signal by WT.

\subsection{Frequency, amplitude, and initial phase estimation}

After applying the wavelet transform to the phase history of range lines, the vibration signal is extracted, which can be expressed in the following form

$$
\tilde{r}_{v 0}(\eta)=\sum_{i=1} \tilde{a}_{i} \sin \left(2 \pi \tilde{f}_{v i} \eta+\tilde{\phi}_{i}\right)
$$

By applying the Fourier transform directly to (19), the spectrum is obtained. It is convenient to get the estimated frequencies $\tilde{f}_{v i}$ from the spectrogram.

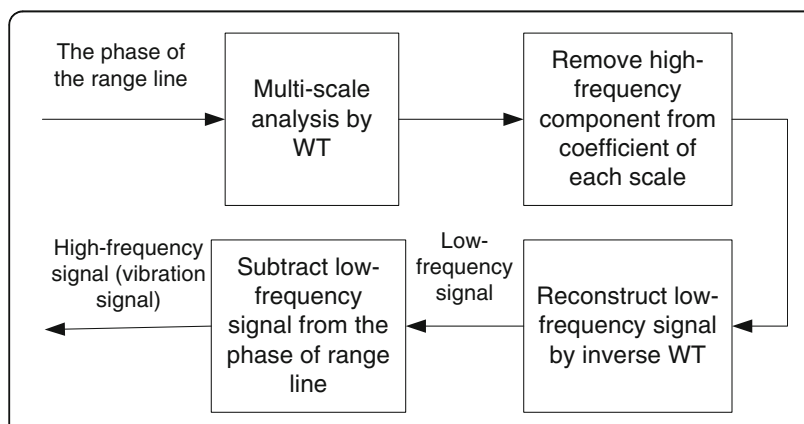

Fig. 5 The algorithm chart of separating signal by WT

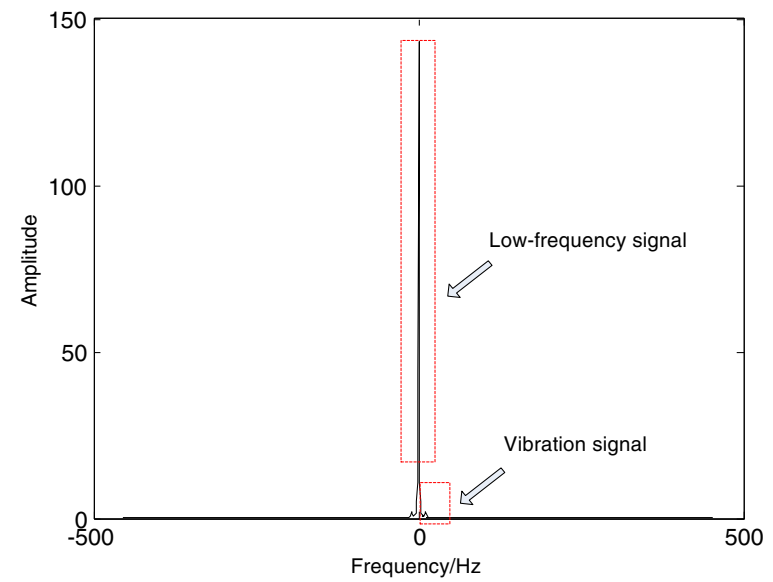

Fig. 6 Spectrum obtained directly by applying FFT to the phase

The amplitudes $\tilde{A}_{i}$ in frequency domain are also easy to obtain. Since the amplitudes in time domain and in frequency domain have the relationship as (20):

$$
\tilde{A}_{i}=\frac{T}{2} / d \eta \cdot \tilde{a}_{i}
$$

where $T$ is the total azimuth time and $d \eta$ is the sample interval in azimuth direction, the estimated amplitudes are acquired.

In order to estimate its initial phase, we define the basis function as follows:

$$
r_{\mathrm{vref}}\left(\eta, \hat{\varphi}_{i}\right)=\tilde{a}_{i} \sin \left(2 \pi \tilde{f}_{v i} \eta+\hat{\varphi}_{i}\right)
$$

where $\hat{\varphi}_{i} \in[0,2 \pi]$. The projection of estimated vibration signal $\tilde{r}_{v 0}(\eta)$ on the basis function can be expressed as

$$
S\left(\hat{\varphi}_{i}\right)=\left\langle\tilde{r}_{\nu 0}(\eta), r_{\mathrm{vref}}\left(\eta, \hat{\varphi}_{i}\right)\right\rangle
$$

The estimated initial phase $\tilde{\varphi}_{i}$ is the $\hat{\phi}_{i}$ in $[0,2 \pi]$ which makes $S\left(\hat{\varphi}_{i}\right)$ achieve the maximum.

\section{Discussions}

In real-world applications, the performance of the proposed method is affected by the presence of noise. If SAR returns are highly corrupted by noise, some errors may exist in the estimated vibration displacement. Thus, we are interested in finding the SNR threshold above which the estimation result is acceptable.

One metric to evaluate the estimation accuracy is the normalized root-mean-square error (NRMSE) [21]. It denotes the normalized Euclidean distance between the estimated vibration signal $\hat{r}_{v 0}(\eta)$ and the real vibration signal $r_{\nu 0}(\eta)$. NRMSE can be calculated by 


$$
\mathrm{NRMSE}=\frac{\left\|\tilde{r}_{v 0}(\eta)-r_{v 0}(\eta)\right\|}{\left\|r_{v 0}(\eta)\right\|}
$$

where $\|\cdot\|$ denotes the $l_{2}$ norm operator.

Adding a zero-mean complex-valued white Gaussian noise to every SAR return, we compute NRMSEs to evaluate the performance of the proposed method in estimating the vibration signal under different SNR levels. Here, we define the SNR as

$$
\mathrm{SNR}=10 \lg \frac{\sigma^{2}}{\sigma_{w}^{2}}
$$

where $\sigma^{2}$ is the power of each return echo and $\sigma_{w}^{2}$ is the variance of the additive noise.

Figure 7 shows an example of the vibration signal estimated by the proposed method. The real vibration signal is assumed to be a sinusoidal wave with a frequency of $20 \mathrm{~Hz}$. Figure $7 \mathrm{~b}$ shows when $\mathrm{SNR}=10 \mathrm{~dB}$, the noise has a little influence on the estimated signal. When SNR decreases to $0 \mathrm{~dB}$, the estimated signal is severely corrupted by the noise.

In order to assess the SNR threshold clearly, we carried out a simulations for three vibration frequencies under different SNRs. The normalized root-mean-square errors are plotted in Fig. 8. The vibration frequencies are 10,20 , and $30 \mathrm{~Hz}$, respectively. When SNR is lower than $-15 \mathrm{~dB}$, the NRMSEs are relatively higher. And with the decrease of SNR, the NRMSEs rise extremely fast. In this case, the estimated frequency may be correct, but it seems not reliable. When SNR is increased to $-15 \mathrm{~dB}$, the NRMSEs drop to an acceptable level. But the errors plateau as the SNR increases. The results also imply that when SNR is larger than $-15 \mathrm{~dB}$, the higher the vibration frequency is, the lower the NRMSEs are.

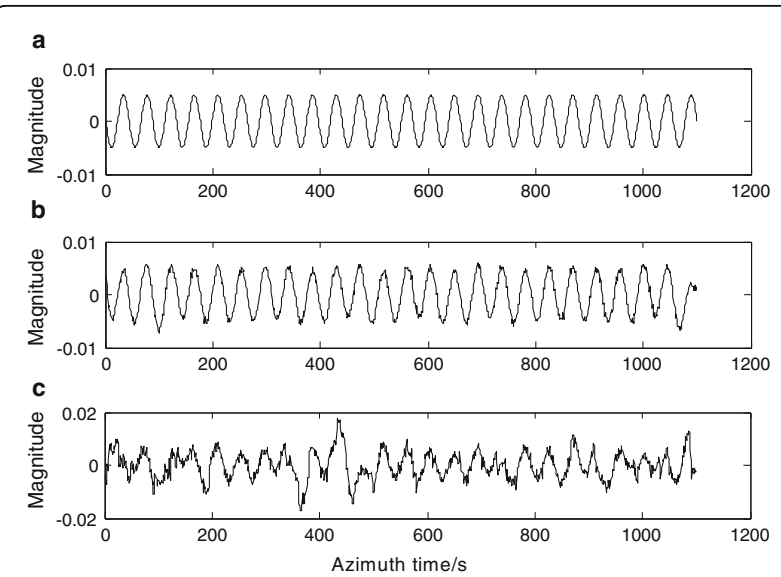

Fig. 7 Vibration signal estimated by phase-analysis method. a Real vibration signal. b Estimated signal with $\mathrm{SNR}=10 \mathrm{~dB}$. c Estimated signal with $\mathrm{SNR}=0 \mathrm{~dB}$

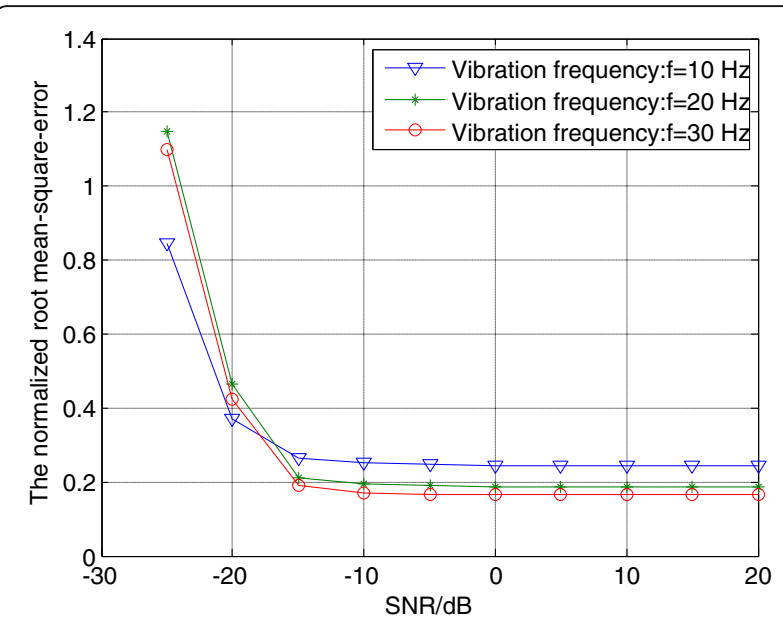

Fig. 8 Normalized root-mean-square errors in estimating vibration signal for three frequencies under different SNRs using phase-analysis-based estimator

\section{Simulations}

\subsection{Airborne radar along desirable trajectory}

\subsubsection{Single point target}

The simulated SAR is an airborne side-looking stripmode SAR working in the $K_{u}$ band. Table 1 lists the key system parameters associated with the simulation. The vibrating target with amplitude of $5 \mathrm{~mm}$, a frequency of $20 \mathrm{~Hz}$, and initial phase of $\pi$ rad is located at $(0,7000,0)$. After applying range compression and range migration correction to the simulated SAR returns, the range line of the vibrating object was obtained. Figure 9a shows the time-frequency representation and the real phase of the vibrating target's range line. Its time-frequency representation illustrates that the small vibration modulates the phase history of the radar echoes. The real phase was acquired by the method proposed in Section 3.1, which consists of two components: the variation with azimuth time of the nominal line-of-sight distance from the target to the radar sensor and the vibration signal, which are referred to as the low-frequency signal and the highfrequency signal, respectively. Figure $9 \mathrm{~b}$ shows the result of the estimation. The first figure is the nominal line-of-

Table 1 SAR system parameters used in the simulation

\begin{tabular}{ll}
\hline Parameter & Quantity \\
\hline Carrier frequency & $15 \mathrm{GHz}$ \\
Bandwidth & $400 \mathrm{MHz}$ \\
Pulse duration & $0.5 \mathrm{\mu s}$ \\
Range sampling frequency & $420 \mathrm{MHz}$ \\
Range resolution & $0.375 \mathrm{~m}$ \\
Plane velocity & $100 \mathrm{~m} / \mathrm{s}$ \\
Length of the synthetic aperture & $125 \mathrm{~m}$ \\
Pulse repetition frequency & $880 \mathrm{~Hz}$ \\
\hline
\end{tabular}


a
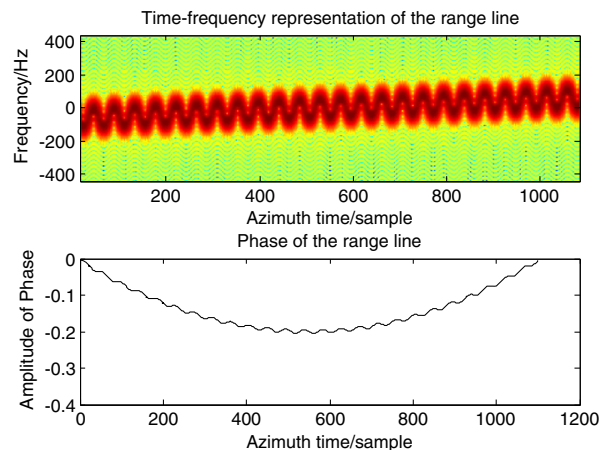

C
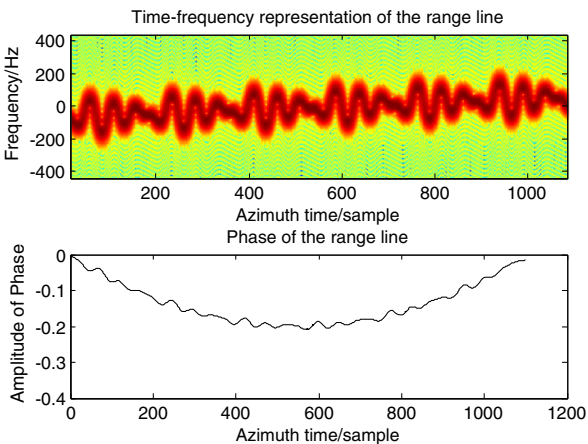

b
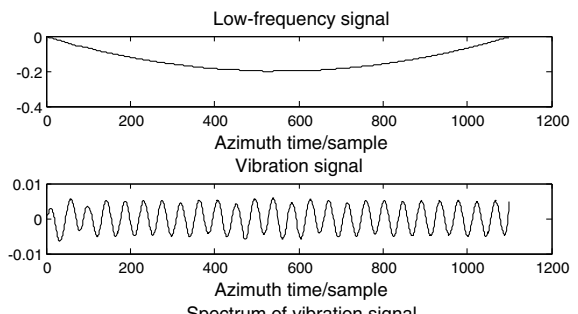

Spectrum of vibration signal

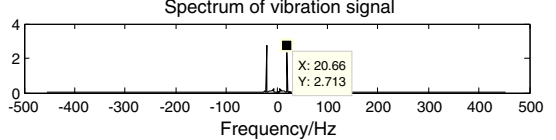

d

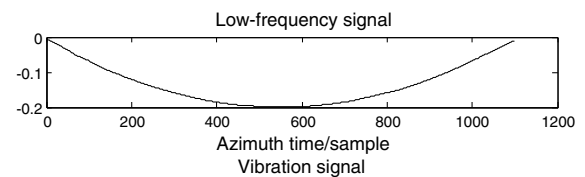
Vibration signal

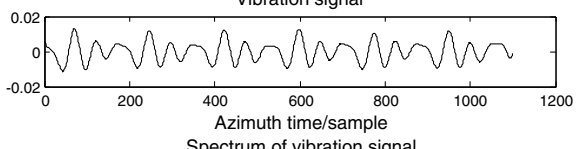

Spectrum of vibration signal

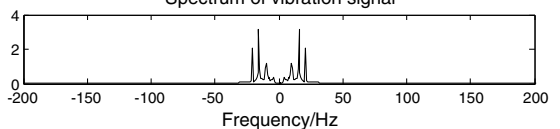

Fig. 9 The simulation results for one vibrating point target. $\mathbf{a}, \mathbf{b}$ Time-frequency representation, the phase of the range line and the vibration estimation results for a target whose vibration signal is a single-frequency sinusoidal. $\mathbf{c}$, $\mathbf{d}$ Time-frequency representation, the phase of the range line and the vibration estimation results for a target whose vibration signal is a multi-frequency sinusoidal

sight distance varied with azimuth time, which is equivalent to a low-frequency signal separated from the phase of the range line by WT. Then subtracting this low-frequency signal from the phase of the range line, we obtained the vibration signal, as shown in the second figure. After applying fast Fourier transform (FFT) to the estimated vibration signal, the vibration frequency was acquired, as illustrated in the last figure. The estimated amplitude and initial phase is $4.9 \mathrm{~mm}$ and $3.0945 \mathrm{rad}$ by the proposed method.

Supposing that the vibration signal is a multi-frequency sinusoidal signal, whose equation is given by

$$
\begin{aligned}
r_{\nu 0}(\eta)= & 0.003 \sin (20 \pi \eta+11 \pi / 9) \\
& +0.006 \sin (30 \pi \eta+\pi) \\
& +0.004 \sin (40 \pi \eta+\pi / 4)
\end{aligned}
$$

Time-frequency representation and phase of the range line is shown in Fig. 9c. It is clear that a multi-frequency signal modulates the phase history of SAR returned signals. Figure $9 \mathrm{~d}$ demonstrates the result of the estimation, from which we know that the estimated vibration signal contains three frequencies. The estimated frequencies are 9.917, 15.7, and $20.66 \mathrm{~Hz}$, which are almost the same as the real frequencies. The estimated amplitudes are
2.1, 5.7, and $3.8 \mathrm{~mm}$ correspondingly and the estimated initial phase is as Table 2 .

\subsubsection{Multiple point targets in different range cells}

Then we considered the case where multiple point targets in different range cells are contained in the scene. Suppose that there are three vibrating targets $O, P$, and $Q$, located at $(0,6990,0),(0,7000,0)$, and $(0,7005,0)$. The vibration amplitude, frequency, and initial phase of $O, P$, and $Q$ are shown in Table 3 . The range lines of the three targets are shown in Fig. 10a. Applying the proposed method to these range lines respectively, we got the results of estimation for point $O, P$, and $Q$, as shown in Fig. 10b-d. The estimated vibration parameters are listed in Table 4, which are almost the same as the corresponding parameters set in Table 3.

Table 2 Comparison of real phase and estimated phase

\begin{tabular}{lccc}
\hline Real phase/rad & $11 \pi / 9(3.8397)$ & $\pi(3.1416)$ & $\pi / 4(0.7854)$ \\
Estimated phase/rad & 3.7856 & 3.3929 & 0.8011 \\
\hline
\end{tabular}


Table 3 Vibration parameters of three targets

\begin{tabular}{llll}
\hline Targets & 0 & $P$ & $Q$ \\
\hline Amplitude/mm & 3 & 6 & 4 \\
Frequency/Hz & 25 & 15 & 20 \\
Initial phase/rad & $2 \pi / 3(2.0944)$ & $\pi(3.1416)$ & $\pi / 4(0.7854)$ \\
\hline
\end{tabular}

\subsection{Airborne radar along undesirable trajectory}

As mentioned in Section 3.1, if the aircraft flies with a velocity $v_{Y}$, the slant range $R(\eta)$ would fluctuate with azimuth time. Suppose that the aircraft's track is a sinusoid, which can be written as

$$
y=\sin (\pi / 62.5 x)
$$

The vibrating target with amplitude of $5 \mathrm{~mm}$ and a frequency of $10 \mathrm{~Hz}$ is located at $(0,7000,0)$. Applying the same method to the simulated SAR returns, we obtained the result as shown in Fig. 11.

In fact, the aircraft's fluctuation frequency is very low so that it is also can be referred to as a low-frequency signal compared to the vibration signal. Hence, the proposed method still holds true in the case of the undesirable trajectory.

\subsection{Comparison with TF analysis method, DFRFT-based method, and HAF-based method}

As described in Section 1, several methods about extracting the vibration signatures with SAR systems have been proposed during the last few years. The time-frequency (TF) analysis method is an important tool to analyze the micro-Doppler effect. DFRFT is an effective method to estimate the time-varying accelerations, frequencies, and displacements associated with vibrating objects [10-12]. Moreover, a time-frequency tracking algorithm based on the high-order ambiguity function (HAF) is employed to reconstruct the instantaneous frequency law of the microDoppler $[16,17]$. In this section, we compare them with phase-analysis method.

5.3.0.1 Time-frequency analysis method The timefrequency distributions to provide methods use timefrequency distributions to provide an analysis of the micro-Doppler effect. The time-frequency representation of the range line is shown in Fig. 12, which roughly reveals a $4-\mathrm{Hz}$ vibration component. However, this is only

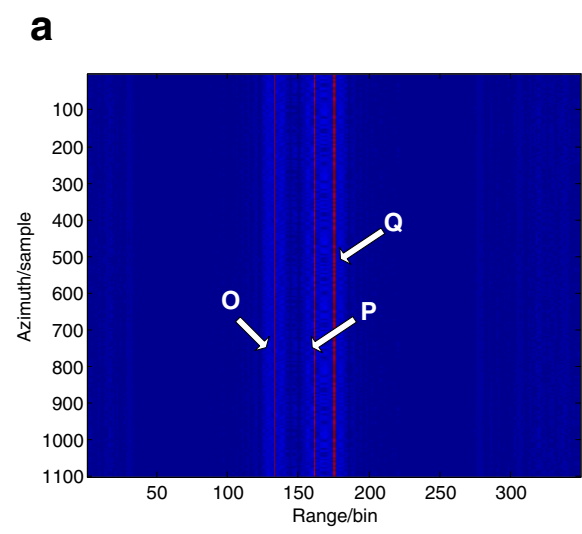

C
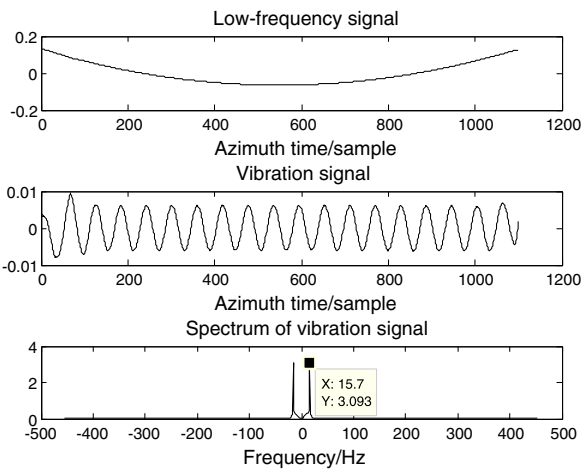

\section{b}

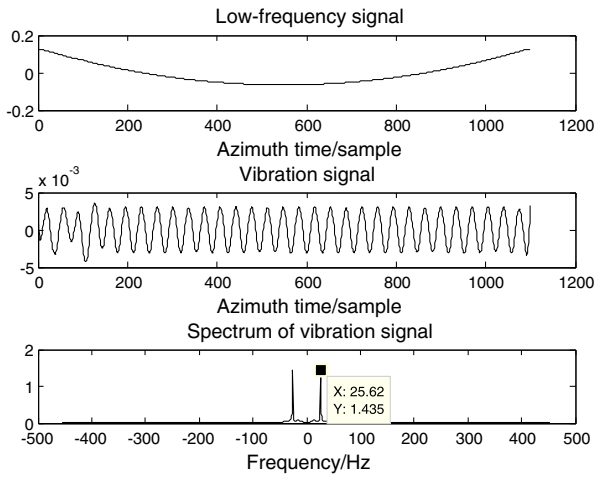

d

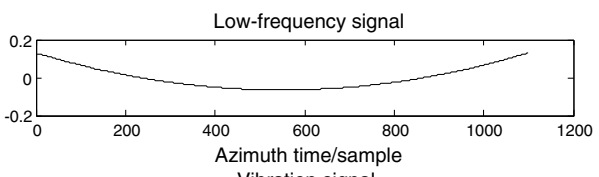

Vibration signal

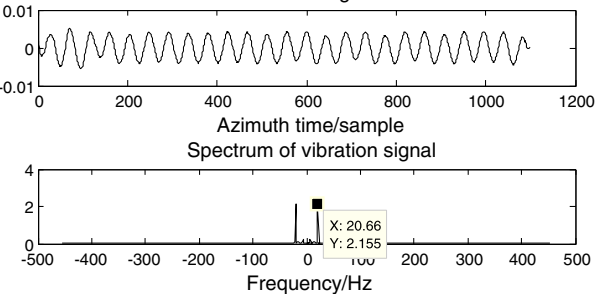

Fig. 10 The simulation results for three vibrating point targets in different range cells. a Shows the range lines of the three targets. $\mathbf{b}-\mathbf{d}$ The results of vibration estimation for target $O, P$, and $Q$ 
Table 4 Estimated vibration parameters of three targets

\begin{tabular}{llll}
\hline Targets & $O$ & $P$ & $Q$ \\
\hline Amplitude/mm & 2.6 & 5.4 & 3.8 \\
Frequency/Hz & 25.62 & 15.7 & 20.66 \\
Initial phase/rad & 2.0577 & 3.2358 & 0.8482
\end{tabular}

a qualitatively deduced observation. To obtain precise estimations of the instantaneous displacement from the time-frequency representation, further estimation procedures are required. In addition, the resolution of timefrequency distribution is closely related to the length of the time window, and we cannot get optimal time resolution and frequency resolution at the same time. If the vibration frequencies are higher, or there are multiple vibration components, the time-frequency representation would not be clear enough to reveal all the vibration components. Whereas the proposed method provides direct quantitative estimations of the vibration displacement and a larger range of the estimated vibration frequency.

5.3.0.2 DFRFT-based method The DFRFT-based method approximates the nonstationary signal by a chirp signal in a small time window. DFRFT is applied to estimate the vibration acceleration. Then the history of the vibration acceleration can be reconstructed and the vibration frequency can be obtained by calculating DFT of the acceleration. The proposed method extracts the phase of the global data directly and applies the WT to the phase history to obtain the vibration signal.

A simulated example is provided to compare the capability of the two methods on vibration signal reconstruction and spectrum estimation. We assumed that the point target's vibration signal is $r_{\nu 0}(\eta)=0.005 \sin (8 \pi \eta)$. The estimation results are shown in Fig. 13. The estimated frequency of DFRFT-based method and phase-analysis method is

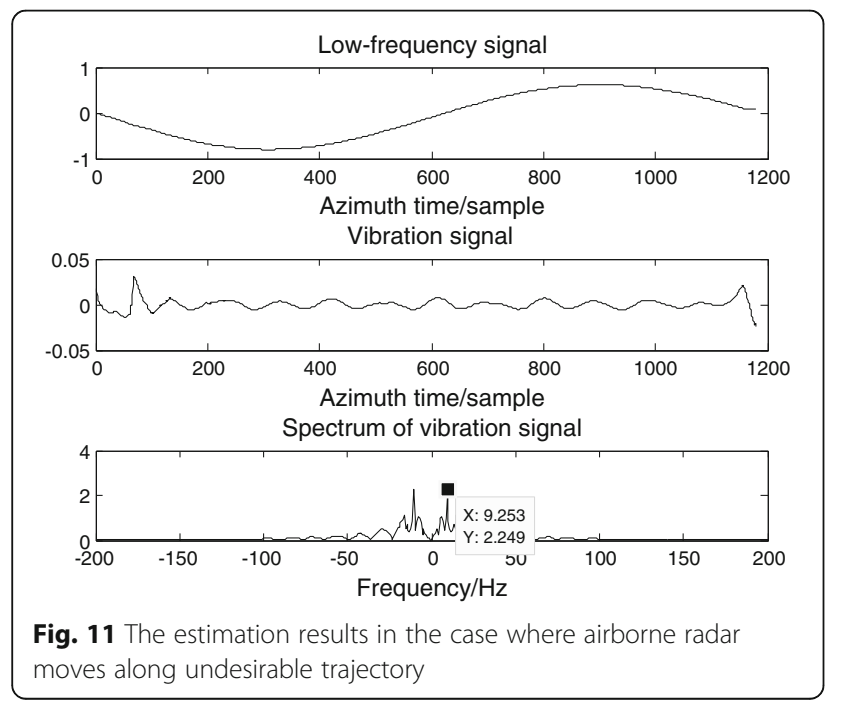

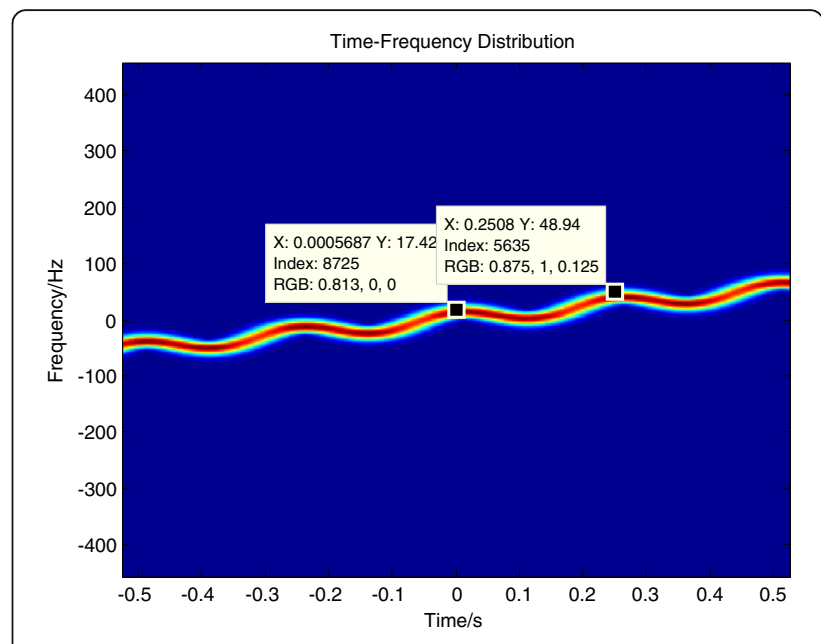

Fig. 12 The time-frequency representation of the range line (the actual vibration frequency is $4 \mathrm{~Hz}$ )
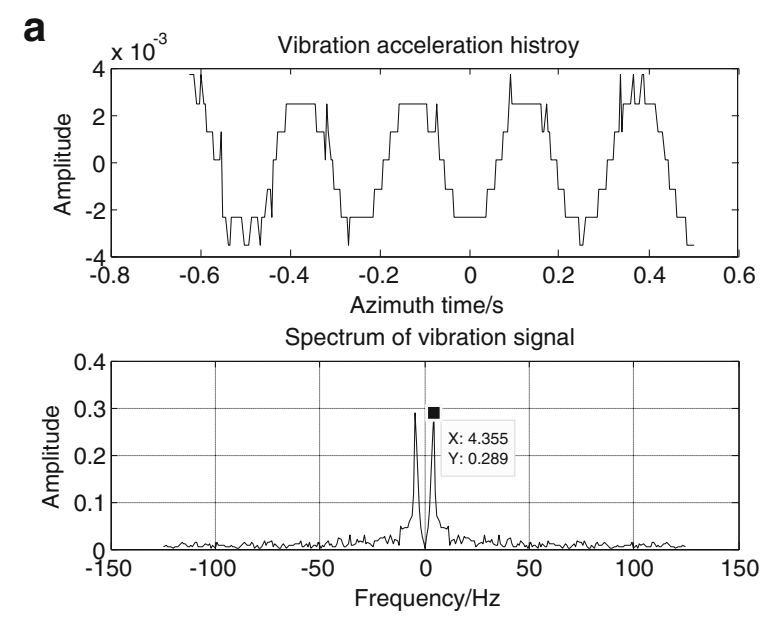

b
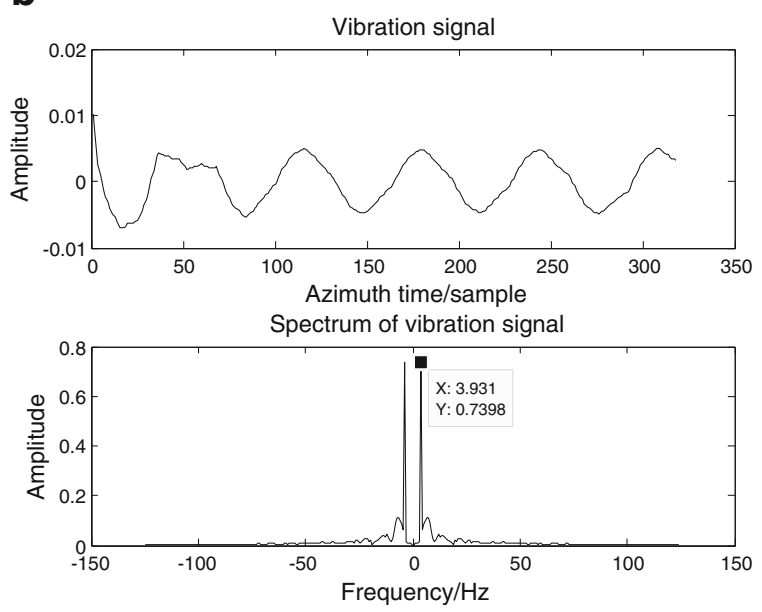

Fig. 13 The estimation results by two different methods. a Obtained by DFRFT-based method. $\mathbf{b}$ Obtained by phase-analysis method 
4.355 and $3.931 \mathrm{~Hz}$, and the computation time is 5.7754 and $0.4108 \mathrm{~s}$, respectively. It demonstrates that the phaseanalysis method does an excellent reconstruction of smooth vibration displacement and provides more accurate estimation results. Furthermore, it also implies that the proposed method in this paper has lower computation complexity.

5.3.0.3 HAF-based method The HAF-based method tracks the instantaneous frequency laws by dividing the range line of the vibrating target in nonoverlapping windows and locally approximating the phase with a polynomial expression. In each window, HAF algorithm is applied, which is an iterative algorithm, to estimate the polynomial coefficients. After the initial reconstruction, the instantaneous frequency laws must be filtered in order to mitigate the possible gaps that may appear at the transitions between estimation windows [17]. On the other hand, the phase-analysis method needs neither iterative algorithm in each window nor filtering for estimated instantaneous frequency laws. Instead, it gives the history of the vibration displacement directly and then the vibration amplitudes, frequencies, and initial phases can be estimated. Therefore, our proposed method may have relatively low computation complexity. Besides, our method makes use of the global data so that the possible errors induced by transitions between the estimation windows may be avoided.

\section{Conclusions}

In this paper, a new method for radar-based vibration estimation was presented, making the radar capable of more accurate vibration measurement. We obtain the SAR phase history by applying the range compression and range migration correction to the SAR returns. Then the wavelet transform is acted on the phase history to extract the vibration displacement. This method provides quantitative estimations of the vibration signature including vibration amplitudes, frequencies, and initial phases. Some simulations provided to demonstrate that it can estimate the vibration signature of one or several targets vibrating in single or multiple frequencies successfully and it also can be used for the case where the aircraft with the radar sensor moves along desirable or undesirable trajectory. Note that in this paper, we assume that the aircraft flies at a constant speed. When the aircraft flies at the speed varied with time, the fluctuation of the velocity would generate a little interference to the phase history, but the vibration signal can be still extracted exactly. Therefore, the influence of the changed velocity can be neglected.

However, this method has limitation that targets have to be distributed in different range cells. We cannot extract the vibration displacement of each target which is in the same range cell. In our future work, the case of multiple point targets in the same range cell and the models of real-world vibrating objects will be examined carefully, which will have great significance to object recognition, classification, and SAR imaging.

\section{Acknowledgements}

This work was supported in part by the National Natural Science Foundation of China under Grant 61201366, in part by the Fundamental Research Funds for the Central Universities under Grant NS2016040, in part by the Fundamental Research Funds for the Central Universities under Grant NJ20150020.

\section{Competing interests}

The authors declare that they have no competing interests.

Received: 3 March 2016 Accepted: 25 August 2016

Published online: 07 September 2016

\section{References}

1. H Wu, M Siegel, P Khosla, Vehicle sound signature recognition by frequency vector principal component analysis. IEEE Trans. Instrum. Meas. 48(5), 1005-1009 (1999)

2. $\mathrm{VC}$ Chen, $\mathrm{F} \mathrm{Li}, \mathrm{S} \mathrm{Ho}, \mathrm{H}$ Wechsler, Micro-Doppler effect in radar: phenomenon, model, and simulation study. IEEE Trans. Aerosp. Elecron. Syst. 42(1), 2-21 (2006)

3. Q Wang, M Pepin, A Wright, R Dunkel, T Atwood, B Santhanam, W Gerstle, AW Doerry, MM Hayat, Reduction of vibration-induced artifacts in synthetic aperture radar Imagery. IEEE Trans. Geosci. Remote Sens. 52(6), 3063-3073 (2014)

4. Y Zhang, JP Sun, P Lei, W Hong, SAR-based paired echo focusing and suppression of vibrating targets. IEEE Trans. Geosci. Remote Sens. 52(12), 7593-7605 (2014)

5. T Sparr, B Krane, Micro-Doppler analysis of vibrating targets in SAR. IEE Proc. Radar, Sonar Navigat. 150(4), 277-283 (2003)

6. T Sparr, B Krane, Analysis of phase modulation caused by target motion in SAR images. Proc. SPIE 5102, 178-188 (2003)

7. T Sparr, B Krane, Time-frequency analysis of vibrating targets in airborne SAR systems. IEE Proc. Radar, Sonar Navigat. 150(3), 173-176 (2003)

8. M. Rüegg, E. Meier, D. Nüesch, Constant Motion, Acceleration, Vibration, and Rotation of Objects in SAR Data. Proc. SPIE 5980, 598005-1-12 (2005)

9. M Rüegg, E Meier, D Nüesch, Vibration and rotation in millimeter-wave SAR. IEEE Trans. Geosci. Remote Sens. 45(2), 245-255 (2007)

10. Q. Wang, M. Pepin, R.J. Beach, R. Dunkel, T. Atwood, A.W. Doerry, B. Santhanam, W. Gerstle, M.M. Hayat, Demonstration of Target Vibration Estimation in Synthetic Aperture Radar Imagery. In Proc. IEEE Int. Geosci. Remote Sens. Symp. (IGARSS, Vancouver, 2011), pp.4083-4086

11. Q Wang, M Pepin, RJ Beach, R Dunkel, T Atwood, B Santhanam, W Gerstle, AW Doerry, MM Hayat, SAR-based vibration estimation using the discrete fractional Fourier transform. IEEE Trans. Geosci. Remote Sens. 50(10), 4145-4156 (2012)

12. A. Jelili, B. Santhanam, M. Hayat, Limitations and Capabilities of the Slanted Spectrogram Analysis Tool for SAR-Based Detection Of Multiple Vibrating Targets. $201448^{\text {th }}$ Asilomar Conference on Signals, Systems and Computers. (Pacific Grove, CA, 2014), pp.172-176

13. B Peng, XZ Wei, B Deng, HW Chen, Z Liu, X Li, A sinusoidal frequency modulation Fourier transform for radar-based vehicle vibration estimation. IEEE Trans. Instrum. Meas. 63(9), 2188-2199 (2014)

14. Y. Zhang, J.P. Sun, Moving Target Vibration Estimation in SAR Using Chirp Modulation and Autofocus. In Proc. IEEE Int. Radar Conf. (RADAR, Lille, 2014), pp.1-6

15. Y Liang, Q Zhang, Y Luo, YQ Bai, YA Chen, Micro-Doppler features analysis and extraction of vibrating target in FWCW SAR based on slow time envelope signatures. IEEE Geosci. Remote Sens. Lett. 12(10), 2041-2045 (2015)

16. A. Anghel, G. Vasile, C. loana, R. Cacoveanu, S. Ciochina, Vibration Estimation in SAR Images Using Azimuth Time-Frequency Tracking and a Matched Signal Transform. In Proc. IEEE Int. Geosci. Remote Sens. Symp. (IGARSS, Milan, 2015), pp.2576-2579

17. A Anghel, G Vasile, C Loana, R Cacoveanu, S Ciochina, Micro-Doppler reconstruction in spaceborne SAR images using azimuth time-frequency tracking of the phase history. IEEE Geosci. Remote Sens. Lett. 13(4), 604-608 (2016)

18. B.P. Lathi, Signal Processing and Linear Systems (Univ. California, Berkeley, 1998) 
19. G. Cumming, F.H. Wong, Digital Processing of Synthetic Aperture Radar Data: Algorithms and Implementation (Publishing House of Electronics Industry, Beijing, 2012), pp. 154-191

20. RK Raney, Synthetic aperture imaging radar and moving targets. IEEE Trans. Aerosp. Electron. Syst. AES-7(3), 499-505 (1971)

21. H. Kaibaf, J.T. Case, Y.R. Zheng, S. Kharkovsky, R. Zoughi, Quantitative and Qualitative Comparison of SAR Images from Incomplete Measurements Using Compressed Sensing and Nonuniform FFT. In Proc. Radar Conf. (RADAR, Kansas City, 2011), pp. 592-596

\section{Submit your manuscript to a SpringerOpen ${ }^{\circ}$ journal and benefit from:}

- Convenient online submission

- Rigorous peer review

- Immediate publication on acceptance

- Open access: articles freely available online

- High visibility within the field

- Retaining the copyright to your article

Submit your next manuscript at $>$ springeropen.com 